NOTICE: this is the author's version of a work that was accepted for publication in Automatica. Changes resulting from the publishing process, such as peer review, editing, corrections, structural formatting, and other quality control mechanisms may not be reflected in this document. Changes may have been made to this work since it was submitted for publication. A definitive version was subsequently published in Automatica, Vol. 49, No. 7 (2013).

DOI: 10.1016/j.automatica.2013.03.008 


\title{
Some new results in the theory of negative imaginary systems with symmetric transfer matrix function *
}

\author{
Augusto Ferrante ${ }^{a}$, Lorenzo Ntogramatzidis ${ }^{b}$ \\ a Dipartimento di Ingegneria dell'Informazione, Università di Padova, via Gradenigo, 6/B - 35131 Padova, Italy. \\ Research partially carried out while visiting Curtin University, Perth (WA), Australia. \\ ${ }^{\mathrm{b}}$ Department of Mathematics and Statistics, Curtin University, Perth (WA), Australia.
}

\begin{abstract}
This note represents a first attempt to provide a definition and characterisation of negative imaginary systems for not necessarily rational transfer functions via a sign condition expressed in the entire domain of analyticity, along the same lines of the classic definition of positive real systems. Under the standing assumption of symmetric transfer function, we then derive a necessary and sufficient condition that characterises negative imaginary transfer functions in terms of a matrix sign condition restricted to the imaginary axis, once again following the same line of argument of the standard positive real case. Using this definition, even transfer functions with a pole at the origin with double multiplicity, as well as with a possibly negative relative degree, can be negative imaginary.
\end{abstract}

Key words: negative imaginary systems, symmetric transfer functions, reciprocal $m$-ports.

\section{Introduction}

Positive real linear systems constitute an important cornerstone of passivity theory. The notion of positive real system was first introduced in electrical network analysis to indicate dissipative $m$-port networks. In the single-input singleoutput case, positive real systems can be realised with electrical circuits involving only resistors, capacitors and inductors. In the multiple-input multiple-output case, $m$-port electrical networks can be realised using also transformers and gyrators, in addition to the above-mentioned electrical components. The most interesting case is by far the one of the so-called reciprocal $m$-ports, which can be realised by resistors, capacitors, inductors and transformers, i.e., without the need for gyrator elements. The interesting feature of reciprocal $m$-port electrical networks is the fact that the corresponding impedance, admittance and scattering matrices are always symmetric. Furthermore, in practice gyrators can only be realised by using active electrical elements. We refer to $[1,3]$ and the references cited therein.

\footnotetext{
^ Partially supported by the Italian Ministry for Education and Research (MIUR) under PRIN grant n. 20085FFJ2Z "New Algorithms and Applications of System Identification and Adaptive Control" and by the Australian Research Council under the grant FT120100604.

Email addresses: augusto@dei.unipd.it (Augusto Ferrante), L. Ntogramatzidis@curtin.edu.au (Lorenzo Ntogramatzidis).
}

The usual approach in establishing a positive real theory is to first introduce the classic definition of positive real function as a (not necessarily rational) complex matrix of functions which is analytic in the right half complex plane and which satisfies, in the domain of analyticity, a certain inequality. For rational functions, the second step usually consists in characterising the concept of positive realness in terms of conditions involving properties of the restriction of the matrix function to the imaginary axis. Another characterisation is given in terms of the celebrated positive real Lemma equations, a cornerstone of modern control theory that have generated an endless stream of literature. Two fairly recent papers whose reference lists points to many of the fundamental contributions and generalisations in this field are $[5,6]$.

A fundamental feature of the classic positive real theory is the fact that the relative degree of rational positive real systems is either zero or plus or minus one, [3]. It was noticed in [9] that, when modelling undamped or lightly damped flexible structures with colocated position sensors and force actuators, the resulting transfer function has a relative degree which is greater than one. This consideration led to the introduction of the so-called negative imaginary systems, which impose a weaker restriction on the relative degree of the transfer function. A subsequent very promising stream of literature flourished in the last few years on the properties of negative imaginary systems, see $[10,17,18,16,15,14,11,12,19]$ and the references cited 
therein.

In the first contribution on this topic [9], a definition was proposed for negative imaginary function matrix in terms of properties of the function on the imaginary axis. In this first definition, systems with poles on the imaginary axis were excluded. This is also the way negative imaginary systems were introduced in the contribution by the same authors in IEEE Control Systems Magazine, [14]. This restriction was abandoned in $[18,16]$, where a simple pole on the imaginary axis except at the origin was allowed. A further important extension of this new negative imaginary theory has subsequently been proposed in order to deal with systems with a single pole at the origin. More recently, also the case of a double pole at the origin was considered, see $[7,13]$. In recent months, several efforts have been devoted to the generalisation and adaptation of this theory to different contexts and scenarios, including implicit systems in [12]. Lossless imaginary systems have been recently introduced in [17] and [19]. The reason for the success of the negative imaginary theory is that many systems that are important and useful in practice are negative imaginary. On the other hand, so far the theory appears to be incomplete as, differently from the positive real case, the definitions of negative imaginary systems have always been restricted to the case of systems with rational transfer function. This paper may be viewed as a very first attempt to propose a general definition (valid for systems having non-rational transfer function) that will hopefully lead to a general comprehensive theory in the same vein of that of positive real systems. In particular, our definition allows for the presence of delays that, on the one hand have non-rational transfer function and, on the other hand, are very often present in real applications.

Our definition is given in terms of a matrix sign condition in the entire domain of analyticity, in the same spirit of the classic positive real case. To that end, the notion of skewimaginary matrix function will be instrumental. Our effort in this direction was motivated by the results on the closed loop stability of systems having (non necessarily rational) positive real transfer function. Therefore, as pointed out by B.D.O. Anderson in [2], it seems natural and potentially very convenient to have a general definition of negative imaginary systems that does not require rationality.

Then, in a way that once again parallels the conventional positive real approach, [1], a necessary and sufficient condition will be introduced for the rational case that characterises a negative imaginary transfer function in terms of its behaviour on the imaginary axis. This alternative approach has the advantage of being applicable to possibly non-proper transfer functions, as well as to transfer functions with more than one pole at the origin, and even to non rational transfer functions. The price to pay is the restriction to symmetric transfer functions. Nevertheless, as aforementioned, the symmetric case is by far the most relevant and interesting in practice, as it is the one that corresponds to reciprocal $m$-port electrical networks. In addition, undamped or lightly damped flexible structures with colocated position sensors and force actuators are characterised by a symmetric transfer function, [14,19].

Notation. Given a matrix $A, A^{\mathrm{T}}$ denotes the transpose of $A$ and $A^{*}$ denotes the complex conjugate transpose of $A$. Given a matrix-valued function $F(s)$, we denote by $F^{*}(s)$ the function $[F(s)]^{*}$.

\section{Positive Real and Negative Imaginary functions: the standard definitions}

We begin by recalling the standard definition of positive real function. For a detailed discussion on positive real systems we refer to $[1,3]$ and the references cited therein.

Definition 2.1 The function $F: \mathbb{C} \longrightarrow \mathbb{C}^{m \times m}$ is positive real if

- $F(s)$ is analytic in the open right half s-plane ( $\mathfrak{R e}\{s\}>0)$;

- $F(s)$ is real when $s$ is real and positive;

- $F(s)+F^{*}(s)$ is positive semi-definite if $\mathfrak{R e}\{s\}>0$.

The following classic result provides necessary and sufficient conditions for a real rational transfer function matrix to be positive real in terms of the features of its restriction to the imaginary axis of the complex plane.

Lemma 2.1 Let $F(s)$ be real and rational. Then $F(s)$ is positive real if and only if

- $F(s)$ has no poles in the open right half s-plane;

- $F(i \omega)+F^{*}(i \omega)$ is positive semi-definite for all real $\omega$ such that $i \omega$ is not a pole of $F(i \omega)$;

- if $i \omega_{0}$ is a pole of any element of $F(s)$, it is a simple pole with Hermitian and positive semidefinite residue. In particular, if $\omega_{0}$ is finite, the residue is

$$
K_{0} \stackrel{\text { def }}{=} \lim _{s \rightarrow i \omega_{0}}\left(s-i \omega_{0}\right) F(s)
$$

while if $\omega_{0}$ is infinite, the residue is

$$
K_{\infty} \stackrel{\text { def }}{=} \lim _{\omega \rightarrow \infty} \frac{F(i \omega)}{i \omega} .
$$

We now present the definition of negative imaginary transfer function in the way it was presented in [16], which constitutes an important extension with respect to the first one given in [9] because, as already observed, does not exclude the case of systems with poles on the imaginary axis (provided they are not at the origin).

Definition 2.2 ([16, Definition 1])

The square real-rational and proper transfer function $G(s)$ is called negative imaginary if

(1) $G(s)$ has no poles at the origin and in $\mathfrak{R e}\{s\}>0$; 
(2) $i\left[G(j \omega)-G^{*}(j \omega)\right] \geq 0$ for all $\omega \in(0, \infty)$ except for the values of $\omega$ where $i \omega$ is a pole of $G(s)$

(3) if $s=i \omega_{0}\left(\right.$ with $\left.\omega_{0} \in(0, \infty)\right)$ is a pole of $G(s)$, then it is a simple pole, and the residual matrix $i K_{0}$ is positive semidefinite Hermitian.

This definition was also generalised in [11] to include the case of transfer function matrices with a single pole at the origin. The following Lemma, which is given in [16], is the analogous of the Positive Real Lemma.

Lemma 2.2 (Negative Imaginary Lemma, [16, Lemma 7]).

Let $(A, B, C, D)$ be a minimal realisation of the $m \times m$ real $r a$ tional proper transfer function matrix $G(s)$, where $A \in \mathbb{R}^{n \times n}$, $B \in \mathbb{R}^{n \times m}, C \in \mathbb{R}^{m \times n}, D \in \mathbb{R}^{m \times m}$. Then, $G(s)$ is negative imaginary if and only if

(1) A is non-singular;

(2) $D$ is symmetric;

(3) a matrix $Y=Y^{\top}>0, Y \in \mathbb{R}^{n \times n}$ exists such that

$$
A Y+Y A^{T} \leq 0 \quad \text { and } B+A Y C^{T}=0
$$

The following theorem establishes a link between the definition of negative imaginary transfer function matrix with the so-called Passivity Theory.

Theorem 2.1 A minimal realisation $(A, B, C, D)$ is negative imaginary if and only if $A$ has no eigenvalues at the origin, $D$ is symmetric and the state equation is dissipative with respect to the supply function

$$
\left[\begin{array}{ll}
x^{T} & u^{T}
\end{array}\right]\left[\begin{array}{ll}
O & S \\
S^{T} & O
\end{array}\right]\left[\begin{array}{l}
x \\
u
\end{array}\right]
$$

where $S=-A^{-T} C^{T}$. The corresponding storage function is $P=\left(A Y A^{T}\right)^{-1}$, where $Y$ satisfies $(1)$.

Proof: First, recall that a reachable state realisation $(A, B)$ is dissipative with respect to the supply function

$$
x^{\mathrm{T}} Q x+2 x^{\mathrm{T}} S u+u^{\mathrm{T}} R u
$$

if and only if the linear matrix inequality

$$
\left[\begin{array}{cc}
Q-A^{\mathrm{T}} P-P A & S-P B \\
S^{\mathrm{T}}-B^{\mathrm{T}} P & R
\end{array}\right] \geq 0
$$

admits a symmetric and positive semidefinite solution $P$, see e.g. [20, page 383].

(Only if). If the system is negative imaginary, $\operatorname{det}(A) \neq$ 0 and (1) holds for a matrix $Y=Y^{\mathrm{T}} \geq 0$. By taking $P=A^{-\mathrm{T}} Y^{-1} A^{-1}$ we find $S-P B=-A^{-\mathrm{T}} C^{\mathrm{T}}-$
$A^{-\mathrm{T}} Y^{-1} A^{-1}\left(-A Y C^{\mathrm{T}}\right)=0$. Moreover,

$$
\begin{aligned}
A^{\mathrm{T}} P+P A & =Y^{-1} A^{-1}+A^{-\mathrm{T}} Y^{-1} \\
& =Y^{-1} A^{-1}\left(Y A^{\mathrm{T}}+A Y\right) A^{-\mathrm{T}} Y^{-1} \leq 0,
\end{aligned}
$$

since $A Y+Y A^{\mathrm{T}} \leq 0$. Therefore

$$
\left[\begin{array}{cc}
-A^{\mathrm{T}} P-P A & S-P B \\
S^{\mathrm{T}}-B^{\mathrm{T}} P & O
\end{array}\right] \geq 0,
$$

which implies that (3) holds with $Q=0$ and $R=0$.

(If). Let $P \geq 0$ be such that (4) holds true. We show that $P$ is invertible. We first prove that $\operatorname{ker} P$ is $A$-invariant. Let

$$
Q=-A^{\mathrm{T}} P-P A .
$$

Take $v \in \operatorname{ker} P$. Then $v^{\mathrm{T}} Q v=v^{\mathrm{T}}\left(-A^{\mathrm{T}} P-P A\right) v=0$, so that $Q v=0$. Then, by post-multiplying (5) by $v$ we get $P A v=0$, which implies that $A v \in \operatorname{ker} P$, i.e. $\operatorname{ker} P$ is $A$-invariant. Now we show that $\operatorname{ker} P \subseteq \operatorname{ker} S^{\mathrm{T}}$. Let $v \in \operatorname{ker} P$. For any $w$ the inequality

$$
0 \leq\left[\begin{array}{ll}
v^{\mathrm{T}} & w^{\mathrm{T}}
\end{array}\right]\left[\begin{array}{cc}
-A^{\mathrm{T}} P-P A & S-P B \\
S^{\mathrm{T}}-B^{\mathrm{T}} P & O
\end{array}\right]\left[\begin{array}{l}
v \\
w
\end{array}\right]
$$

holds since $\left[\begin{array}{cc}-A^{\mathrm{T}} P-P A & S-P B \\ S^{\mathrm{T}}-B^{\mathrm{T}} P & O\end{array}\right] \geq 0$. Then, developing the products, we get $v^{\mathrm{T}} S w+w^{\mathrm{T}} S^{\mathrm{T}} v=2 v^{\mathrm{T}} S w=0$. For the arbitrariness of $w$, we obtain $S^{\mathrm{T}} v=0$. Then, $v \in \operatorname{ker} S^{\mathrm{T}}$. It follows that $\operatorname{ker} P$ is $A$-invariant and is contained in nullspace of $S^{\mathrm{T}}$. Then, it is also contained in the unobservable subspace of the pair $\left(A, S^{\mathrm{T}}\right)$. Therefore, it is contained in the unobservable subspace of $(A, C)$. However, since the realisation is minimal, such unobservable subspace is zero. Therefore, $\operatorname{ker} P=\{0\}$, which means that $P$ is invertible. Now we can define $Y=\left(A^{\mathrm{T}} P A\right)^{-1}$. It is now a matter of following the same steps of the (only if) part backwards to see that $Y$ satisfies (1).

Remark. We have shown that the state equation of a negative imaginary system is dissipative. It is an interesting open problem to understand whether (or under what condition) there exists a supply function such that the input-output relation of a negative imaginary system is dissipative with respect to such a function.

\section{A general definition}

In this section we propose a new definition of negative imaginary system for transfer functions which are not necessarily rational. In this definition, similarly to what happens for the classic positive real case, the sign condition holds in the entire domain of analyticity of the transfer function. We recall that in this paper we restrict our attention to symmetric matrix transfer functions. This is the most relevant situation, 
because it encompasses both the scalar case, and the case of a transfer function of a reciprocal $m$-port electrical network. As already observed, the only way to obtain a nonsymmetric transfer function of an $m$-port electrical network is to employ gyrators, whose physical implementation requires the use of active components but that cannot be physically implemented with arbitrary precision. Furthermore, the transfer functions from a force actuator to a corresponding collocated position sensor (for instance, a piezoelectric sensor) in a lightly damped or undamped structure is typically symmetric, see $[10,14,19]$.

Definition 3.1 Let $G: \mathbb{C} \longrightarrow \mathbb{C}^{m \times m}$ be analytic in an open subset $\Omega$ of $\mathbb{C}$. Then, $G$ is skew-imaginary in $\Omega_{1} \subseteq \Omega$ if

- $i\left[G(s)-G^{*}(s)\right] \geq 0$ for all $s \in \Omega_{1}$ such that $\mathfrak{I m}\{s\}>0$;

- $i\left[G(s)-G^{*}(s)\right]=0$ for all $s \in \Omega_{1}$ such that $\operatorname{Im}\{s\}=0$;

- $i\left[G(s)-G^{*}(s)\right] \leq 0$ for all $s \in \Omega_{1}$ such that $\mathfrak{I m}\{s\}<0$.

Definition 3.2 Let $G: \mathbb{C} \longrightarrow \mathbb{C}^{m \times m}$ be a real, symmetric transfer function. We say that $G$ is symmetric negative imaginary if

- $G(s)$ is analytic in $\mathfrak{R e}\{s\}>0$;

- $G(s)$ is skew-imaginary in $\mathfrak{R e}\{s\}>0$.

Notice that since $G(s)$ is symmetric, $i\left[G(s)-G^{*}(s)\right]=$ $-2 \mathfrak{I m}\{G(s)\}$.

As noted above, with respect to the definitions of negative imaginary transfer functions given in [9], [11] and [16], here we restrict our attention to the symmetric ones. On the other hand, a definition given directly in terms of a sign property of the transfer matrix function in its domain of analyticity appears to be very appealing since it goes in the direction, suggested in [2], of an abstract foundation in the same spirit of the definition of positive real functions. In particular, in this definition, there is no need to start with rational and proper functions as in [9], [11] and [16] or to deal with poles. Notice that this definition may immediately be applied to non-proper rational transfer functions. For example, consider $G(s)=a s$, where $a \in \mathbb{R}$. Then, $G(s)$ is analytic in $\mathfrak{R e}\{s\}>0$ and, by defining $s=\sigma+i \omega$, we get

$i\left[G(s)-G^{*}(s)\right]=i[a \sigma+i a \omega-(a \sigma-i a \omega)]=-2 a \omega$,

which is non-negative for all $\omega \geq 0$ if and only if $a \leq 0$. It follows that $G(s)$ is negative imaginary if and only if $a \leq 0$.

Moreover, the fact, introduced in [7] as an ad hoc extension, that a transfer function with a double pole in the origin may be negative imaginary is also a direct consequence of this general definition. For example, $G(s)=1 / s^{2}$ is easily seen to be negative imaginary. Indeed, defining $s=\sigma+i \omega$, in the domain of analyticity $(\sigma>0)$ the quantity

$$
i\left[G(s)-G^{*}(s)\right]=\frac{4 \sigma \omega}{\left(\sigma^{2}-\omega^{2}\right)^{2}+4 \sigma^{2} \omega^{2}}
$$

is positive definite for all $\omega \geq 0$.

Finally, to the best of our knowledge, this is the first definition that allows the notion of negative imaginary system to be applicable to non-rational transfer functions. In the following example, we consider a system with a delay.

Example. Let $G(s)=-s\left(e^{-s T}+1\right)$, with $T \in \mathbb{R}_{+}$being a positive delay. We now show that $G(s)$ is negative imaginary. Let $s=\sigma+i \omega$. A direct calculation yields

$i\left(G(s)-G^{*}(s)\right)=2\left[\omega+e^{-\sigma T}(\omega \cos (\omega T)-\sigma \sin (\omega T))\right]$.

Now define

$$
f(\sigma, \omega) \stackrel{\text { def }}{=} \omega+e^{-\sigma T}(\omega \cos (\omega T)-\sigma \sin (\omega T)) .
$$

Since $f(\sigma, 0) \equiv 0$, and $f(\sigma,-\omega)=-f(\sigma, \omega)$, we only need to show that, when $\sigma>0$ and $\omega>0, f(\sigma, \omega) \geq 0$. Assume by contradiction that $\bar{\sigma} \geq 0$ and $\omega_{0}>0$ exist such that $f\left(\bar{\sigma}, \omega_{0}\right)<0$. It is immediate to see that $f\left(\sigma, \omega_{0}\right)$, as a function of $\sigma$, is continuos with all its derivatives. Hence, there exists $\sigma_{0}$ such that $\inf _{\sigma \geq 0} f\left(\sigma, \omega_{0}\right)=\min _{\sigma \geq 0} f\left(\sigma, \omega_{0}\right)=$ $f\left(\sigma_{0}, \omega_{0}\right) \leq f\left(\bar{\sigma}, \omega_{0}\right)<0$ and $\frac{\partial f}{\partial \sigma}\left(\sigma_{0}, \omega_{0}\right)=0$. By computing this derivative, we see that the only candidate $\sigma_{0}$ is given by $\sigma_{0}=\frac{1}{T}+\omega_{0} \frac{\cos \left(\omega_{0} T\right)}{\sin \left(\omega_{0} T\right)}$. To get a contradiction, it is now sufficient to show that if $\omega_{0}$ is such that $\sigma_{0}$ is finite and positive, then $f\left(\sigma_{0}, \omega_{0}\right)>0$. Indeed, $f\left(\sigma_{0}, \omega_{0}\right)=$ $\frac{1}{T}\left[\omega_{0} T-e^{-\sigma_{0} T} \sin \left(\omega_{0} T\right)\right]$, which is clearly positive for all $\omega_{0}>0, \sigma_{0}>0$ and $T>0$.

We now specialise the notion of negative imaginary function to real and rational transfer function matrices.

Recall that given a real rational function $G(s)$ and a simple pole $p \in \mathbb{C}$ of $G(s)$, we have a unique decomposition $G(s)=$ $G_{1}(s)+A /(s-p)$, where $G_{1}(s)$ is a rational function which is analytic in an open set containing $p$ and the (non-zero) matrix $A$ is the residue corresponding to the pole $p$. If $p$ is a double pole of $G(s)$, we have the unique decomposition $G(s)=G_{1}(s)+A_{1} /(s-p)+A_{2} /(s-p)^{2}$, where the matrix $A_{1}$ is the residue corresponding to the pole $p$. In this case, by analogy, we define the (non-zero) matrix $A_{2}$ to be the quadratic residue corresponding to the pole $p$. If $G(s)$ has a pole at infinity, it can be uniquely decomposed as $G(s)=$ $G_{1}(s)+P(s)$, where $G_{1}(s)$ is a rational proper function and $P(s)=\sum_{i=1}^{k} A_{i} s^{i}$ is a homogeneous polynomial in $s$. We refer to $A_{i}$ as the $i$-th coefficient in the expansion at infinity of $G(s)$.

Lemma 3.1 Let $G(s)$ be real, symmetric and rational. Then $G(s)$ is symmetric negative imaginary if and only if

(i) $G(s)$ has no poles in $\mathfrak{R e}\{s\}>0$;

(ii) $i\left[G(i \omega)-G^{*}(i \omega)\right] \geq 0$ for all $\omega \in(0, \infty)$ except for the values of $\omega$ where $i \omega$ is a pole of $G(s)$; 
(iii) if $s=i \omega_{0}$, with $\omega_{0} \in(0, \infty)$, is a pole of $G(s)$, then it is a simple pole and the corresponding residual matrix ${ }^{1}$ $K_{0}=\lim _{s \rightarrow i \omega_{0}}\left(s-i \omega_{0}\right) i G(s)$ is Hermitian and positive semidefinite;

(iv) if $s=0$ is a pole of $G(s)$, then it is at most a double pole. Moreover, both its residual and its quadratic residual (when present) are positive semidefinite Hermitian matrices;

(v) if $s=\infty$ is a pole of $G(s)$, then it is at most a double pole. Moreover, both the coefficients in the expansion at infinity of $G(s)$ are negative semidefinite Hermitian matrices.

Proof: Assume that the real and rational matrix function $G(s)$ is negative imaginary. Then $G(s)$ is analytic in the open right half plane, so that (i) is immediate. Secondly, if $\omega>0$ and $i \omega$ is not a pole of $G(s)$, then $G(s)$ is analytic in an open set containing $i \omega$, so that $i\left[G(i \omega)-G^{*}(i \omega)\right]=$ $\lim _{\sigma \searrow 0} i\left[G(\sigma+i \omega)-G^{*}(\sigma+i \omega)\right] \geq 0$, which proves (ii). In order to prove (iii), assume, by contradiction, that $\omega>0$ and $i \omega$ is a pole of $G(s)$ having multiplicity $\mu>1$. Then $G(s)=$ $\frac{A}{(s-i \omega)^{\mu}}+G_{1}(s)$ with $A \neq 0$ and $G_{1}(s)$ such that $\lim _{s \rightarrow i \omega}(s-$ $i \omega)^{\mu} G_{1}(s)=0$. Let $\varepsilon>0$ and $v$ be a complex vector such that $v^{*} A v=M \exp (i \varphi)$ with $M>0$ and $\varphi \in[0,2 \pi)$. Let $s=$ $i \omega+\varepsilon \exp (i \vartheta)$. Then, for all $\vartheta \in[-\pi / 2, \pi / 2]$ and for all sufficiently small $\varepsilon>0$ we have:

$$
\begin{aligned}
0 \leq \varepsilon^{\mu} i v^{*}\left[G(s)-G^{*}(s)\right] v= & -2 M \sin (\varphi-\mu \vartheta) \\
& +\varepsilon^{\mu} i v^{*}\left[G_{1}(s)-G_{1}^{*}(s)\right] v .
\end{aligned}
$$

The second term of the sum in the right-hand side of (6) tends to zero as $\varepsilon$ does. Moreover, if $\mu>1$, for any $\varphi$ we can select $\vartheta_{0} \in[-\pi / 2, \pi / 2]$ in such a way that $\varphi-\mu \vartheta_{0}=\pi / 2$, which gives $\sin \left(\varphi-\mu \vartheta_{0}\right)=1$. Then, for all sufficiently small $\varepsilon>0$ we have:

$$
0 \leq-2 M+f(\varepsilon)
$$

where $f(\varepsilon) \stackrel{\text { def }}{=} \varepsilon^{\mu} i v^{*}\left[G_{1}\left(i \omega+\varepsilon \exp \left(i \vartheta_{0}\right)\right)-G_{1}^{*}(i \omega+\right.$ $\left.\left.\varepsilon \exp \left(i \vartheta_{0}\right)\right)\right] v$. Since, $M>0$ and, as already observed, $\lim _{\varepsilon \rightarrow 0} f(\varepsilon)=0$, (7) is a contradiction. Hence, $\mu$ cannot be larger than 1. Assume now that $\omega>0$ and $i \omega$ is a simple pole of $G(s)$. Then (6) still holds with $\mu=1$. This implies $\sin (\varphi-\vartheta) \leq 0$ for any $\vartheta \in(-\pi / 2, \pi / 2)$, so that $\varphi=-\pi / 2$. Then for any complex vector $v$, the term $v^{*} i A v$ is real and non-negative. Equivalently, $K_{0}=i A$ is Hermitian and positive semidefinite.

Let us now prove (iv). As before, assume, by contradiction, that 0 is a pole of $G(s)$ having multiplicity $\mu>2$. Then $G(s)=\frac{A}{s^{\mu}}+G_{1}(s)$ with $A \neq 0$ and $G_{1}(s)$ such that $\lim _{s \rightarrow 0} s^{\mu} G_{1}(s)=0$. Let $\varepsilon>0$ and $v$ be a complex vector such that $v^{*} A v \neq 0$. Let $M>0$ and $\varphi \in[0,2 \pi)$ be such that $v^{*} A v=M \exp (i \varphi)$. Let $s=\varepsilon \exp (i \vartheta)$. Then, for all

\footnotetext{
1 Notice that $K_{0}$ is the product of the imaginary unit $i$ by the
} residue in $\omega_{0}$. $\vartheta \in[0, \pi / 2]$ and $\varepsilon>0$ we have:

$$
\begin{aligned}
0 \leq \varepsilon^{\mu} i v^{*}\left[G(s)-G^{*}(s)\right] v= & -2 M \sin (\varphi-\mu \vartheta) \\
& +\varepsilon^{\mu} i v^{*}\left[G_{1}(s)-G_{1}^{*}(s)\right] v .
\end{aligned}
$$

Again, the second term of the sum in the right-hand side of (8) tends to zero as $\varepsilon$ does. Moreover, if $\mu>2$, for any $\varphi$ we can select $\vartheta_{0} \in[0, \pi / 2]$ in such a way that that $\sin (\varphi-$ $\left.\mu \vartheta_{0}\right) \geq \sqrt{2} / 2$. Then, for all $\varepsilon>0$ sufficiently small we have:

$$
0 \leq-\sqrt{2} M+f(\varepsilon)
$$

where $f(\varepsilon) \stackrel{\text { def }}{=} \varepsilon^{\mu} i v^{*}\left[G_{1}\left(\varepsilon \exp \left(i \vartheta_{0}\right)\right)-G_{1}^{*}\left(\varepsilon \exp \left(i \vartheta_{0}\right)\right)\right] v$. As before, this leads to a contradiction. Thus, $\mu$ cannot be larger than 2. Assume now that 0 is a double pole of $G(s)$. Then (8) still holds with $\mu=2$. This implies $\sin (\varphi-2 \vartheta) \leq 0$ for any $\vartheta \in(0, \pi / 2)$, yielding $\varphi=0$. Then, for any complex vector $v$, the term $v^{*} A v$ is real and non-negative. Equivalently, $A$ is Hermitian and positive semidefinite. Assume now $G(s)=\frac{A_{2}}{s^{2}}+\frac{A_{1}}{s}+G_{1}(s)$, where $A_{2}$ is Hermitian and positive semidefinite (we allow also the possibility $A_{2}=0$, so that also the case of a simple pole in zero is taken into account) and $\lim _{s \rightarrow 0} s G_{1}(s)=0$. For all $\varepsilon>0$ we have:

$$
0=\varepsilon i\left[G(\varepsilon)-G^{*}(\varepsilon)\right]=i\left(A_{1}-A_{1}^{*}\right)+\varepsilon i\left[G_{1}(\varepsilon)-G_{1}^{*}(\varepsilon)\right],
$$

which, in view of $\lim _{s \rightarrow 0} s G_{1}(s)=0$, implies $A_{1}=A_{1}^{*}$. It remains to show that $A_{1}$ is positive semidefinite. To this end, let $\varepsilon_{1}>0$ and $\varepsilon_{2} \stackrel{\text { def }}{=} \arcsin \left(\varepsilon_{1}^{2}\right)>0$. Moreover, given an arbitrary complex vector $v$, let $M_{i} \stackrel{\text { def }}{=} v^{*} A_{i} v, i=1,2$ (notice that $M_{i}$ are real numbers $)$. Finally, let $s \stackrel{\text { def }}{=} \varepsilon_{1} \exp (i(\pi / 2-$ $\left.\left.\varepsilon_{2}\right)\right)$. Then, for all sufficiently small $\varepsilon_{1}>0$ we have:

$$
\begin{aligned}
0 & \leq \varepsilon_{1} i v^{*}\left[G(s)-G^{*}(s)\right] v \\
& =2 \cos \left(\varepsilon_{2}\right)\left[M_{1}+2 \varepsilon_{1} M_{2}+\frac{\varepsilon_{1}}{\cos \left(\varepsilon_{2}\right)} i v^{*}\left[G_{1}(s)-G_{1}^{*}(s)\right] v\right],
\end{aligned}
$$

which implies $M_{1} \geq 0$, so that $A_{1}$ is positive semidefinite.

The proof of ( $\mathbf{v})$ is very similar to the proof of (iv). As before, assume, by contradiction, that $\infty$ is a pole of $G(s)$ having multiplicity $\mu>2$. Then $G(s)=A s^{\mu}+G_{1}(s)$ with $A \neq 0$ and $G_{1}(s)$ such that $\lim _{s \rightarrow \infty} \frac{1}{s^{\mu}} G_{1}(s)=0$. Let $R>0$ and $v$ be a complex vector such that $v^{*} A v=M \exp (i \varphi)$ with $M>0$ and $\varphi \in[0,2 \pi)$. Let $s \stackrel{\text { def }}{=} R \exp (i \vartheta)$. Then, for all $\vartheta \in[0, \pi / 2]$ and for all sufficiently large $R>0$ we have:

$$
\begin{aligned}
0 \leq \frac{1}{R^{\mu}} i v^{*}\left[G(s)-G^{*}(s)\right] v= & -2 M \sin (\varphi+\mu \vartheta) \\
& +\frac{1}{R^{\mu}} i v^{*}\left[G_{1}(s)-G_{1}^{*}(s)\right] v .
\end{aligned}
$$

The second term of the sum in the right-hand side of (12) tends to zero as $R$ tends to infinity. Now the same argument 
employed in the proof of (iv) allows to conclude that $\mu$ cannot be larger than 2. Assume now that $\infty$ is a double pole of $G(s)$. Then (12) still holds with $\mu=2$. This implies that $\sin (\varphi+2 \vartheta) \leq 0$ for any $\vartheta \in(0, \pi / 2)$, which gives $\varphi=$ $\pi$. Then for any complex vector $v$, the term $v^{*} A v$ is real and non-positive. Equivalently, $A$ is Hermitian and negative semidefinite. Assume now $G(s)=A_{2} s^{2}+A_{1} s+G_{1}(s)$, where $A_{2}$ is Hermitian and negative semidefinite (we allow also the possibility $A_{2}=0$ so that also the case of a simple pole in $\infty$ is taken into account) and $\lim _{s \rightarrow \infty} \frac{1}{s} G_{1}(s)=0$. For all $\varepsilon>0$ we have:

$$
0=\frac{1}{R} i\left[G(R)-G^{*}(R)\right]=A_{1}-A_{1}^{*}+\frac{1}{R} i\left[G_{1}(R)-G_{1}^{*}(R)\right],
$$

which, in view of $\lim _{s \rightarrow \infty} \frac{1}{s} G_{1}(s)=0$, implies $A_{1}=A_{1}^{*}$. It remains to show that $A_{1}$ is negative semidefinite. To this end, let $R>0$ (sufficiently large) and $\varepsilon \stackrel{\text { def }}{=} \arcsin \left(1 / R^{2}\right)>0$. Moreover, given an arbitrary complex vector $v$, let $M_{i} \stackrel{\text { def }}{=}$ $v^{*} A_{i} v, i=1,2$ (notice that $M_{i}$ are real numbers). Finally, let $s \stackrel{\text { def }}{=} R \exp (i(\pi / 2-\varepsilon))$. Then, for all sufficiently large $R>0$ we have:

$$
\begin{aligned}
0 & \leq \frac{i}{R} v^{*}\left[G(s)-G^{*}(s)\right] v \\
& =2 \cos (\varepsilon)\left[-M_{1}-\frac{2}{R} M_{2}+\frac{1}{R \cos (\varepsilon)} i v^{*}\left[G_{1}(s)-G_{1}^{*}(s)\right] v\right]
\end{aligned}
$$

which implies $M_{1} \leq 0$, i.e., $A_{1}$ is negative semidefinite.

Conversely, assume that $G(s)$ is real symmetric and rational and that it satisfies (i) to (v). We have to show that for any given vector $v \in \mathbb{C}^{m}$, the function $i v^{*}\left[G(s)-G^{*}(s)\right] v$ (which in view of (i) is well defined in $\mathfrak{R e}\{s\}>0$ ) is non-negative in the whole open upper-right quarter of the complex plane (the conditions on the positive real axis and on the lowerright quarter of the complex plane are clearly met since $G(s)$ is assumed to be real).

Consider the following closed curve $\gamma$ :

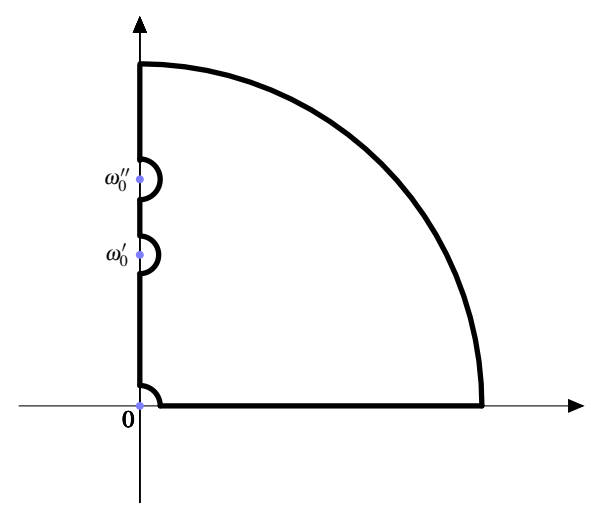

where the small semicircles and the small quarter of a circle, when present, are centred in the (finitely many) purely imaginary poles of $v^{*}\left[G(s)-G^{*}(s)\right] v$ and have radius $\varepsilon$ (if $v^{*}\left[G(s)-G^{*}(s)\right] v$ has no poles in the origin, then the pieces of $\gamma$ in the real and in the imaginary axes are connected through the origin). The radius of the large quarter of a circle centred in the origin is $R$. Now notice that the poles of $v^{*}\left[G(s)-G^{*}(s)\right] v$ are a subset of the poles of $G(s)$. If $i \omega_{0}$ is a pole of $G(s)$ but not of $v^{*}\left[G(s)-G^{*}(s)\right] v$, assumption (ii) and continuity imply that $v^{*}\left[G\left(i \omega_{0}\right)-G^{*}\left(i \omega_{0}\right)\right] v$ is well defined and non-negative.

Let $K$ be the compact set formed by the curve $\gamma$ together with the set that it encircles. For any $\varepsilon>0$ and $R>0, K$ is a compact subset of the domain of analyticity $\Omega$ of $G(s)$. For any given vector $v \in \mathbb{C}^{m}$ and $s \in \Omega$, the function $v^{*} G(s) v$ is analytic in the open right half plane. Thus, its imaginary part $\mathfrak{I m}\left[v^{*} G(s) v\right]=\frac{1}{2} \cdot v^{*}\left[-i\left[G(s)-G^{*}(s)\right]\right] v$ is a harmonic function and therefore, by the maximum principle, $\mathfrak{I m}\left[v^{*} G(s) v\right]$ restricted to $K$ attains its maximum and minimum on the boundary of $K$, i.e., on the curve $\gamma$. Now notice that any given point in the open right half plane belongs to $K$ for a sufficiently large $R$ and a sufficiently small $\varepsilon$. Therefore, it is sufficient to show that for any $v \in \mathbb{C}^{m}$ and $\kappa>0$, there exist $\tilde{R}$ and $\tilde{\varepsilon}$ such that for any $R>\tilde{R}, \varepsilon \in(0, \tilde{\varepsilon})$, and $s \in \gamma$, $i v^{*}\left[G(s)-G^{*}(s)\right] v>-\kappa$. To this aim, let us first observe that:

1. For $s$ in the intersection between $\gamma$ and the real axis, since $G(s)$ is real and symmetric, we have that $s_{0} \in \mathbb{R}$ implies $G\left(s_{0}\right) \in \mathbb{R}^{m \times m}$ and $G^{*}\left(s_{0}\right)=G^{\mathrm{T}}\left(s_{0}\right)=G\left(s_{0}\right)$, so that $G\left(s_{0}\right)-$ $G^{*}\left(s_{0}\right)=0$ and hence $i v^{*}\left[G(s)-G^{*}(s)\right] v=0$.

2. For $s$ in the intersection between $\gamma$ and the imaginary axis, we have $i\left[G(s)-G^{*}(s)\right] \geq 0$ by assumption (ii).

3. For $s$ in the large quarter of a circle, let $G(s)=A_{2} s^{2}+$ $A_{1} s+G_{1}(s)$ with $G_{1}(s)$ proper, where the Hermitian and negative semidefinite matrices $A_{1}$ and $A_{2}$ can also vanish to encompass the cases when $G(s)$ is proper or has a simple pole at infinity. If at least one of the two real numbers $v^{*} A_{2} v$ and $v^{*} A_{1} v$ is nonzero, then it is easy to see that assumption (v) implies that, for $R$ sufficiently large, $i v^{*}\left[G(s)-G^{*}(s)\right] v>0$; if, instead, $v^{*} A_{2} v=v^{*} A_{1} v=0$, then $i v^{*}\left[G(s)-G^{*}(s)\right] v=i v^{*}\left[G_{1}(s)-G_{1}^{*}(s)\right] v$. Since $G(s)$ is symmetric, $G_{1}(s)-G_{1}^{*}(s)$ is strictly proper. Hence, for any given $v$, the quantity $i v^{*}\left[G_{1}(s)-G_{1}^{*}(s)\right] v$ tends to 0 as $R$ tends to infinity. Thus, for any $\kappa>0$, there exists $\tilde{R}$ such that for any $R>\tilde{R}$, and $s=R \exp (j \vartheta)$, with $\vartheta \in[0, \pi / 2]$, it is found that $i v^{*}\left[G_{1}(s)-G_{1}^{*}(s)\right] v>-\kappa$.

4. For $s$ in the small semicircles (when present), consider a semicircle centred in $i \omega_{0}$. The presence of this semicircle implies that $v^{*}\left[G(s)-G^{*}(s)\right] v$ has a pole in $i \omega_{0}$, i.e. that $G(s)=A /\left(s-i \omega_{0}\right)+G_{1}(s)$, where $v^{*} A v \neq 0$ and $G_{1}(s)$ is analytic in an open set containing $i \omega_{0}$. Then, employing assumption (iii), it is easy to check that for $s$ in the small semicircle centred in $i \omega_{0}$, we get $i v^{*}\left[G(s)-G^{*}(s)\right] v \geq 0$ provided that $\varepsilon$ is sufficiently small. 
5. For $s$ in the small quarter of a circle (when present) we have that $G(s)=\frac{A_{2}}{s^{2}}+\frac{A_{1}}{s}+G_{1}(s)$ where $G_{1}(s)$ is analytic in an open set containing 0 . As before, we have that at least one of the terms $v^{*} A_{2} v$ and $v^{*} A_{1} v$ is non-zero. Then, invoking assumption (iv), it is easy to check that for $s$ in the small quarter of a circle centred in 0 , we find $i v^{*}\left[G(s)-G^{*}(s)\right] v \geq$ 0 provided that $\varepsilon$ is sufficiently small.

We now present a result that establishes a relationship between positive real and symmetric negative imaginary rational transfer functions. In particular, the result in Theorem 3.1 parallels [11, Lemma 1] and [13, Lemma 1] obtained for definitions of negative imaginary systems allowing for one or two poles at the origin, respectively, to the definition of symmetric negative imaginary system given in this paper. Before establishing this result, we need the following technical lemma.

Lemma 3.2 $F(s)$ is a real rational positive real function if and only if it may be written as

$$
F(s)=(1 / s) A+F_{1}(s)
$$

where $A$ is symmetric and positive semi-definite and $F_{1}(s)$ is a real rational positive real function with no poles at the origin.

Proof: If $F(s)$ has the form (15) then it is clearly positive real in view of Lemma 2.1. Conversely, assume that $F(s)$ is positive real. If $F(s)$ has no poles at the origin, we may take $F_{1}(s)=F(s)$ and $A=0$ and we have the required decomposition. If $F(s)$ has a pole at the origin, this pole is simple and with symmetric and positive definite residue $A$, in view of Lemma 2.1. Then, we can decompose $F(s)$ in the form (15) where $A$ is indeed the residue at the origin and has therefore the prescribed properties and $F_{1}(s)$ is a real rational function without poles at the origin. It remains to show that $F_{1}$ is itself positive real. To this end, we use again Lemma 2.1. Since $F(s)$ is positive real, it is analytic on the open right half-plane and hence $F_{1}(s)$ has the same property. Moreover, if $i \omega_{0}$ is a pole of $F_{1}(s)$ with multiplicity $\mu$ and residue $A_{0}$, then it is also a pole of $F(s)$ with the same multiplicity and the same residue. Then, $\mu=1$, and $A_{0}$ is symmetric and positive semi-definite. The same holds for a possible pole of $F_{1}(s)$ at infinity. Finally, notice that for $\omega \neq 0$ such that $i \omega$ is not a pole of $F_{1}$ we have $F(i \omega)+F^{*}(i \omega)=F_{1}(i \omega)+F_{1}^{*}(i \omega)$ so that $F_{1}(i \omega)+F_{1}^{*}(i \omega)$ is Hermitian and positive semi-definite. Since $F_{1}(s)+F_{1}^{*}(s)$ is analytic at the origin, we also have that $F_{1}(0)+F_{1}^{*}(0)$ is Hermitian and positive semi-definite.

Theorem 3.1 Let $G(s)$ be a real, rational and proper symmetric negative imaginary matrix transfer function. Then $F(s) \stackrel{\text { def }}{=} s[G(s)-G(\infty)]$ is positive real. Conversely, let $F(s)$ be symmetric, real rational and positive real matrix transfer function. Then $G(s) \stackrel{\text { def }}{=}(1 / s) F(s)+D$ is symmetric negative imaginary for any symmetric matrix $D$.
Proof: Let $G(s)$ be a symmetric, real rational and proper negative imaginary matrix transfer function. We have to show that $F(s) \stackrel{\text { def }}{=} s[G(s)-G(\infty)]$ is positive real. It is clear that $F(s)$ is analytic in the open right half plane and has no pole at infinity. Moreover, $F$ and $G$ have the same poles with the possible exception of the pole at the origin. Let $\omega_{0}>0$ be given and assume that $i \omega_{0}$ is not a pole of $G(s)$. Then, it is not a pole of $F(s)$ and, taking into account that $G^{*}(\infty)=G^{\mathrm{T}}(\infty)=G(\infty)$, we have:

$$
\begin{aligned}
& F\left(i \omega_{0}\right)+F^{*}\left(i \omega_{0}\right) \\
& \quad=i \omega_{0}\left[G\left(i \omega_{0}\right)-G(\infty)\right]-i \omega_{0}\left[G^{*}\left(i \omega_{0}\right)-G^{*}(\infty)\right] \\
& \quad=i \omega_{0}\left[G\left(i \omega_{0}\right)-G^{*}\left(i \omega_{0}\right)\right]+i \omega_{0}\left[G^{\mathrm{T}}(\infty)-G(\infty)\right] \\
& \quad=\omega_{0}\left(i\left[G\left(i \omega_{0}\right)-G^{*}\left(i \omega_{0}\right)\right]\right) \geq 0
\end{aligned}
$$

where the last inequality is due to the fact that $\omega_{0}>0$ and $G$ is negative imaginary. Assume now that $\omega_{0}>0$ is such that $i \omega_{0}$ is a pole of $G(s)$. Then, it is necessarily a simple pole of $G(s)$, and it is also a simple pole of $F(s)$. We then have $G(s)=\frac{A}{s-i \omega_{0}}+G_{1}(s)$ and $K_{0} \stackrel{\text { def }}{=} i A$ is Hermitian and positive semidefinite. Let us compute the residue of $F(s)$ in $i \omega_{0}$. This is given by:

$$
\begin{aligned}
& \lim _{s \rightarrow i \omega_{0}}\left(s-i \omega_{0}\right) F(s)= \\
& \quad \lim _{s \rightarrow i \omega_{0}} s A+\left(s-i \omega_{0}\right)\left[G_{1}(s)-G(\infty)\right]=\omega_{0} i A \geq 0 .
\end{aligned}
$$

Let us consider now the case when $\omega_{0}<0$. If $i \omega_{0}$ is not a pole of $G(s)$ and $F(s)$, we have $F\left(i \omega_{0}\right)+F^{*}\left(i \omega_{0}\right)=$ $\overline{F\left(-i \omega_{0}\right)+F^{*}\left(-i \omega_{0}\right)}$ which is positive semi-definite since it is the complex conjugate of $F\left(-i \omega_{0}\right)+F^{*}\left(-i \omega_{0}\right)$, which we have already seen to be positive semi-definite. A similar argument can be used to show that when $i \omega_{0}$ is a pole of $G(s)$ and $F(s)$, its residue as a pole of $F$ is the complex conjugate of the residue of the pole of $F$ in $-i \omega_{0}$, and hence is positive semi-definite.

Let us consider now the case when $\omega_{0}=0$. Let us first assume that $G(s)$ has no pole at the origin. In this case $F(s)$ has no pole at the origin and $F(0)=0$, so that $F(0)+$ $F^{*}(0)=0 \geq 0$. If $G(s)$ has a simple pole at the origin, then $F(s)$ has no pole at the origin. Moreover, $G(s)=A / s+G_{1}(s)$ where $A$ is positive semi-definite. Thus $F(0)+F^{*}(0)=A+$ $A^{\mathrm{T}}=2 A \geq 0$. If $G(s)$ has a double pole at the origin, $G(s)=$ $A_{2} / s^{2}+A_{1} / s+G_{1}(s)$, where $A_{2} \geq 0$. Hence $F(s)=A_{2} / s+$ $A_{1}+s\left(G_{1}(s)-G(\infty)\right)$. Since $G_{1}(s)$ is analytic in an open set containing the origin, also $A_{1}+s\left(G_{1}(s)-G(\infty)\right)$ is analytic in such an open set. Thus $F(s)$ has a simple pole at the origin and the corresponding residue $A_{2}$ is positive semi-definite.

Conversely, let $F(s)$ be symmetric, real rational and positive real matrix transfer function and $G(s) \stackrel{\text { def }}{=}(1 / s) F(s)+D$. As long as $D=D^{\mathrm{T}}$, the matrix $D$ is clearly irrelevant for the definition of negative imaginary, and it is sufficient to show that $G_{0}(s) \stackrel{\text { def }}{=}(1 / s) F(s)$ is negative imaginary. To this 
end, we first observe that $G_{0}(s)$ is a proper rational function and it is analytic in the open right half-plane. Moreover, $F(s)$ and $G_{0}(s)$ have the same poles with the possible exception of the pole at the origin. Let $\omega_{0}>0$ be given and assume that $i \omega_{0}$ is not a pole of $F(s)$. Then it is not a pole of $G_{0}(s)$. Moreover, $i\left[G_{0}\left(i \omega_{0}\right)-G_{0}^{*}\left(i \omega_{0}\right)\right]=i\left[\left(1 / i \omega_{0}\right) F\left(i \omega_{0}\right)+\right.$ $\left(1 / i \omega_{0}\right) F^{*}\left(i \omega_{0}\right)=\left(1 / \omega_{0}\right)\left[F\left(i \omega_{0}\right)+F^{*}\left(i \omega_{0}\right)\right]$ which is positive semi-definite because $\omega_{0}>0$ and $F$ is positive real. Let now $\omega_{0}>0$ be given and assume that $i \omega_{0}$ is a pole of $F(s)$ and hence of $G_{0}(s)$. Since $F(s)$ is positive real $i \omega_{0}$ is a simple pole of $F(s)$ and hence of $G_{0}(s)$. Moreover, the residue of $i \omega_{0}$ as a pole of $F(s)$ is a positive semi-definite matrix $A$. It is now evident that $\lim _{s \rightarrow i \omega_{0}}\left(s-i \omega_{0}\right) i G_{0}(s)=\left(1 / \omega_{0}\right) A$ which is clearly positive semi-definite.

The case of a pole at the origin is more delicate. If $F(0)=0$ then $G_{0}(s)$ does not have a pole at the origin and this concludes the proof. If $F(s)$ does not have a pole at the origin but $F(0) \neq 0$, then $G_{0}(s)$ has a simple pole at the origin and its residue is $F(0)$ which is positive semi-definite because $F$ is positive real. In the case when $F$ has a simple pole at the origin we use Lemma 3.2 and decompose $F(s)=(1 / s) A+F_{1}(s)$ with $A$ symmetric and positive semidefinite and $F_{1}(s)$ positive real. Hence, $F_{1}(0)$ is positive semi-definite. Thus, $G_{0}(s)$ has a double pole at the origin, whose residue is $F_{1}(0) \geq 0$ and whose quadratic residue is $A \geq 0$.

Remark 3.1 As aforementioned, the difference between the above result and Lemma 1 in [11] is that we allow a double pole at the origin for negative imaginary transfer functions. This is very natural for two reasons: (i) positive real functions can have a single pole at the origin (the most common example being $F(s)=1 / s$ which is easily seen to be positive real) so that it is natural to allow a double pole at the origin for negative imaginary transfer functions. In fact, it is easily seen that $F(s)=1 / s$ satisfies Definition 1 in [11] and is therefore positive real (and this is extremely intuitive since $F(s)$ is the transfer function of a capacitor). Hence, in view of Lemma 1 in [11] we should have that $G(s)=1 / s^{2}$ is negative imaginary which is, again, intuitive. However, $G(s)=1 / s^{2}$ is not negative imaginary according to the Definition 2 in [11]. (ii) Since $G(s)=1 /\left(s^{2}+\varepsilon^{2}\right)$ is negative imaginary for any $\varepsilon \neq 0$, it is natural to require, by continuity, that also $G(s)=1 / s^{2}$ is negative imaginary. We observe that the above result is in line with Lemma 1 in [13], in which the definition of negative imaginary system was extended to include systems with a double pole at the origin.

\section{Concluding remarks}

In this paper we presented a definition of symmetric negative imaginary system that hinges entirely on properties of the transfer function matrix, and not on properties of a finitedimensional realisation. Thus, with the approach presented here even non rational transfer functions - which correspond to infinite-dimensional realisations - can turn out to be negative imaginary. We have then characterised negative imag- inary rational transfer functions by an algebraic condition and established a connection between negative imaginary and positive real transfer functions.

\section{References}

[1] B.D.O. Anderson, and S. Vongpanitlerd. Network Analysis and Synthesis: a Modern Systems Theory Approach. Upper Saddle River, NJ: Prentice Hall, 1973.

[2] B.D.O. Anderson. Comment after the plenary presentation by I. $R$. Petersen at the first Australian Control Conference, Melbourne, Australia, Nov. 10, 2011.

[3] B. Brogliato, R. Lozano, B. Maschke and O. Egeland. Dissipative Systems Analysis and Control Theory and Applications. 2nd ed. New York: Springer-Verlag, 2007.

[4] C. Cai, and G. Hagen. Stability analysis for a string of coupled stable subsystems with negative imaginary frequency response. IEEE Transactions on Automatic Control, AC-55:1958-1963, 2010.

[5] A. Ferrante. Positive real lemma: necessary and sufficient conditions for the existence of solutions under virtually no assumptions. IEEE Trans. Automat. Contr. Vol. AC-50(5):720-724, 2005.

[6] A. Ferrante. Minimal representations of continuous-time processes having spectral density with zeros in the extended imaginary axis. System \& Control Letters. Vol. 54(5):511-520, Apr. 2005.

[7] M. A. Mabrok, A. G. Kallapur, I. R. Petersen and A. Lanzon Stabilisation of conditional uncertain negative-imaginary systems using Riccati equation approach In Proceedings of the 20th International Symposium on Mathematical Theory of Networks and Systems (MTNS). Melbourne, July 9-13, 2012.

[8] A. Lanzon, and I.R. Petersen. "A modified positive-real type stability condition". In Proceedings of the European Control Conference 2007, pp. 3912-3918, Kos, Greece, Jul. 2007.

[9] A. Lanzon, and I.R. Petersen. Stability robustness of a feedback interconnection of systems with negative imaginary frequency response. IEEE Transactions on Automatic Control, AC-53(4):10421046, 2008

[10] Z. Song, A. Lanzon, S. Patra, and I.R. Petersen. "Robust performance analysis for uncertain negative-imaginary systems". International Journal of Robust and Nonlinear Control, vol. 22(3), pp. 262-281, Feb 2012

[11] M.A. Mabrok, A.G. Kallapur, I.R. Petersen, and A. Lanzon. "Stability analysis for a class of negative imaginary feedback systems including an integrator". In Proceedings of the $8^{\text {th }}$ Asian Control Conference 2011, Kaohsiung, Taiwan, May 15-18, 2011.

[12] M.A. Mabrok, A.G. Kallapur, I.R. Petersen, and A. Lanzon "Generalized negative imaginary lemma for descriptor systems", Journal of Mechanics Engineering and Automation, vol. 2(1), pp. 1721, Jan 2012

[13] M.A. Mabrok, A.G. Kallapur, I.R. Petersen, and A. Lanzon. "A stability result on the feedback interconnection of negative imaginary systems with poles at the origin". In Proceedings of the $2^{\text {nd }}$ Australian Control Conference 2012, pp. 98-103, Sydney, November 15-16, 2012.

[14] I. R. Petersen and A. Lanzon. Feedback control of negative imaginary systems. IEEE Control System Magazine, vol. 30, no. 5, pp. 54-72, 2010.

[15] Z. Song, A. Lanzon, S. Patra, and I.R. Petersen. Towards controller synthesis for systems with negative imaginary frequency response. IEEE Transactions on Automatic Control, AC-55:1506-1511, 2010.

[16] J. Xiong, I.R. Petersen, and A. Lanzon. A negative imaginary lemma and the stability of interconnections of linear negative imaginary systems. IEEE Transactions on Automatic Control, AC-55(10):23422347, 2010. 
[17] J. Xiong, I.R. Petersen, and A. Lanzon. "On lossless negative imaginary systems". In Proceedings of the $7^{\text {th }}$ Asian Control Conference 2009, pp. 824-829, Hong Kong, Aug 2009.

[18] J. Xiong, I.R. Petersen, and A. Lanzon. "Finite frequency negative imaginary systems". IEEE Transactions on Automatic Control, In press, DOI 10.1109/TAC.2012.2193705.

[19] J. Xiong, I.R. Petersen, and A. Lanzon. On lossless negative imaginary systems. Automatica, 48:1213-1217, 2012.

[20] J. C. Willems. Dissipative dynamical systems - Part II: Linear systems with quadratic supply rates Archive for Rational Mechanics and Analysis Vol. 45, pp. 352-393, 1972 